\title{
A Prognostic Model to Facilitate Palliative Care Referral in Oncology Outpatients
}

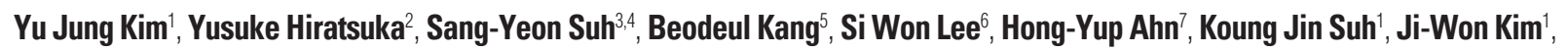 \\ Se Hyun Kim ${ }^{1}$, Jin Won Kim ${ }^{1}$, Keun-Wook Lee ${ }^{1}$, Jee Hyun Kim ${ }^{1}$, Jong Seok Lee ${ }^{1}$ \\ ${ }^{1}$ Division of Hematology and Medical Oncology, Department of Internal Medicine, Seoul National University Bundang Hospital, Seoul National \\ University College of Medicine, Seongnam, Korea, ${ }^{2}$ Department of Palliative Medicine, Tohoku University School of Medicine, Sendai, Japan, \\ ${ }^{3}$ Hospice \& Palliative Care Center, Department of Family Medicine, Dongguk University Ilsan Hospital, Goyang, ${ }^{4}$ Department of Medicine, \\ Dongguk University Medical School, Seoul, ${ }^{5}$ Division of Medical Oncology, CHA Bundang Medical Center, CHA University School of Medicine, \\ Seongnam, ${ }^{6}$ Division of Medical Oncology, Department of Internal Medicine, Yonsei Cancer Center, Yonsei University College of Medicine, Seoul, \\ ${ }^{7}$ Department of Statistics, Dongguk University Medical School, Seoul, Korea
}

Purpose We aimed to develop a prognostic model to assist palliative care referral at least 3 months before death in advanced cancer patients treated at an outpatient medical oncology clinic.

Materials and Methods In this prospective cohort study, a total of 200 patients were enrolled at a tertiary cancer center in South Korea. The major eligibility criterion was an expected survival of less than a year as estimated by their oncologists. We analyzed the influences of known prognostic factors along with chemotherapy status, mid-arm circumference, and triceps skinfold thickness on survival time.

Results The mean age of the patients was 64.5 years, $36 \%$ were female, and the median survival time was 7.6 months. In the multivariate analysis, we found six significant factors related to poor survival: a poor Eastern Cooperative Oncology Group (ECOG) performance status $(\geq 2)$, not undergoing chemotherapy, anorexia, a low lymphocyte level (<12\%), a high lactate dehydrogenase (LDH) level ( $\geq 300 \mathrm{IU} / \mathrm{L})$, and a low mid-arm circumference $(<23 \mathrm{~cm})$. We developed a prognostic model (score, 0-8.0) to predict 3-month survival based on the multivariate analysis. Patients who scored $\geq 4.0$ points had a short survival of less than 3 months ( $p<0.001)$. The discriminating ability of the prognostic model using the area under the receiver operating characteristic curve was 0.88 .

Conclusion The prognostic model using ECOG performance status, chemotherapy status, anorexia, lymphocytes, LDH, and mid-arm circumference can predict 3-month survival in medical oncology outpatients. It can alert oncologists to refer patients to palliative care specialists before it is too late.

Key words Neoplasms, Prognosis, Survival, Palliative care

\section{Introduction}

Several randomized studies have shown that early palliative care referral can improve quality of life, satisfaction with care, and even survival in advanced cancer patients [1-3]. Therefore, many guidelines including the American Society of Clinical Oncology practice guidelines, strongly recommend dedicated palliative care services concurrent with active anticancer treatment [4].

Although there is no doubt regarding the need for and benefits of early palliative care, the real-world status may be far from ideal due to a lack of resources and other barriers. In the real world, many patients do not have the opportunity to receive hospice care at the end of life. The rates of hospice utilization in the United States and the United Kingdom are $50.7 \%$ and $44 \%[5,6]$, respectively. In South Korea, only $22.9 \%$ of advanced cancer patients were referred to government- certified hospice centers in 2018 [7].

Recently, referral to palliative care specialists might be increasing [8], but many patients are still not referred or referred too late in tertiary cancer centers. In one study, $45 \%$ of decedents in the cohort at a comprehensive cancer center had a palliative care consultation and their median survival after the consultation was 1.4 months [9]. Among these patients, $33 \%$ were referred earlier ( $>3$ months before death), and those patients showed improved end-of-life care compared with those who were referred late $(\leq 3$ months before death) [10]. According to international consensus, many experts considered a prognosis of 3-12 months as appropriate timing to refer an advanced cancer patient to a palliative care specialist, and a prognosis of less than 3 months was considered too late or much too late [11].

In the era of targeted therapy and immunotherapy, the treatment decision is becoming more complex, and more

Correspondence: Sang-Yeon Suh

Department of Medicine, Dongguk University Medical School, 123 Dongdae-ro, Gyeongju 38066, Korea

Tel: 82-31-961-7490 Fax: 82-31-961-7969 E-mail: lisasuhmd@hotmail.com

Received April 20, 2021 Accepted July 10, 2021 Published Online July 12, 2021

*Yu Jung Kim and Yusuke Hiratsuka contributed equally to this work. 
patients are receiving anticancer treatment near the end of life. Consequently, some medical oncologists may often hesitate to refer patients to palliative care or lose the appropriate timing to refer patients to palliative care $[12,13]$.

The objective of this study was to develop a prognostic model to assist palliative care referral at least 3 months before death in advanced cancer patients treated at outpatient medical oncology clinics.

\section{Materials and Methods}

\section{Patients}

We enrolled advanced cancer patients treated at a comprehensive cancer center of a university hospital in this prospective cohort study from March 2016 to January 2019. Patients were eligible if they had a diagnosis of advanced cancer, when their oncologists estimated their survival to be less than a year and were 18 years or older. We defined advanced cancer as a metastatic or recurrent disease or progressive locally advanced disease not amenable to curative treatment. Patients were excluded if they had hematologic malignancies, were expected to survive less than a month, and were not able to communicate.

\section{Data collection}

The patients were interviewed face to face by a clinical research nurse after enrollment. They were questioned about the presence of dyspnea, dysphagia, anorexia, edema, fatigue, and weight loss (more than 10\% weight loss over 6 months). Patient performance status was assessed by the Eastern Cooperative Oncology Group (ECOG) performance status and Karnofsky Performance Scale (KPS). We obtained demographic data and clinical information including primary tumor site, anticancer treatment, date of diagnosis or recurrence, date of advanced cancer diagnosis, and date of diagnosis as incurable. The results of laboratory tests were collected from electronic medical records. A trained registered nurse measured triceps skinfold thickness and midarm circumference of each patient. Cutoff levels for triceps skinfold thickness and mid-arm circumference were determined arbitrarily based on the 25 th percentile level of healthy Korean adults [14].

\section{Statistical analysis}

Descriptive analyses were performed to summarize the baseline characteristics of the patients. Survival time was calculated using the Kaplan-Meier method. We performed univariate analyses to identify significant prognostic factors using the log-rank test. We included age, sex, and variables with a p-value less than 0.05 in univariate analyses in the multivariate analysis. A stepwise regression analysis was performed using the Cox proportional hazards model to identify independent prognostic factors. Based on the results of the final model, a risk score for each prognostic factor was calculated by dividing the $\beta$ coefficient of the variable by the lowest $\beta$ coefficient in the model. The sum of the partial scores for each patient was calculated, which ranged from 0 to 8.0. We determined the cutoff points for the prediction of survival times shorter than 3 months to obtain the highest accuracy. The discriminatory ability of the risk score was measured using the area under the receiver operating characteristic (ROC) curve. All tests were two-sided, and $\mathrm{p}$-values $<0.05$ were considered significant. Confidence intervals (CIs) were calculated at a 95\% confidence level. All analyses were performed using JMP ver. 14 for Windows (SAS Institute Inc., Cary, NC).

\section{Results}

We enrolled 200 patients with advanced cancer who had visited the outpatient medical oncology clinic at a comprehensive cancer center of a university hospital between March 2016 and January 2019 (Table 1). The mean patient age was 64.4 years (standard deviation, 11.6 years; range, 32 to 85 years), and 128 patients $(64 \%)$ were male. In total, $66 \%$ of the patients had an ECOG performance status of 0 or 1, and $90 \%$ of them had a KPS of $70 \%$ or more. Sixty-five percent of patients were undergoing palliative chemotherapy at the time of enrollment. The most common cancer types were lung $(33.5 \%)$, genitourinary tract $(14.5 \%)$, colorectal $(14 \%)$, and stomach cancer $(10 \%)$. The median time from the diagnosis of advanced cancer to enrollment was 14.1 months. With a median follow-up period of 7.7 months, 159 patients died, and the median overall survival time was 7.6 months (95\% CI, 1.3 to 33.3).

In the univariate analyses, statistically significant poor prognostic factors were a poor ECOG performance status $(\geq 2)$; not undergoing chemotherapy; the presence of dyspnea; anorexia; edema; fatigue; leukocytosis; anemia; lymphopenia; a low albumin level; a high level of aspartate transaminase, lactate dehydrogenase $(\mathrm{LDH})$, or C-reactive protein; a low mid-arm circumference $(<23 \mathrm{~cm})$; and a low triceps skinfold thickness $(<0.8 \mathrm{~cm}$ ) (Table 2$)$. In the multivariate analysis, six factors including a poor ECOG performance status $(\geq 2)$, not undergoing chemotherapy, the presence of anorexia, a low lymphocyte level $(<12 \%)$, a high $\mathrm{LDH}$ level $(\geq 300 \mathrm{IU} / \mathrm{L})$, and a low mid-arm circumference $(<23 \mathrm{~cm})$ were independently associated with a poor prognosis (Table 3). Factors with collinearity were excluded from the final multivariate analysis. 
Table 1. Patient characteristics

\begin{tabular}{|c|c|}
\hline Characteristic & No. $(\%)(n=200)$ \\
\hline Age (yr) & $64.4 \pm 11.6$ \\
\hline \multicolumn{2}{|l|}{ Sex } \\
\hline Male & $128(64.0)$ \\
\hline Female & $72(36.0)$ \\
\hline \multicolumn{2}{|l|}{ Primary cancer site } \\
\hline Lung & $67(33.5)$ \\
\hline Stomach & $20(10.0)$ \\
\hline Colon/Rectal & $28(14.0)$ \\
\hline Breast & $18(9.0)$ \\
\hline Ovary/Cervical & $4(2.0)$ \\
\hline Liver/Biliary tract & $4(2.0)$ \\
\hline Pancreas & $4(2.0)$ \\
\hline Esophagus & $5(2.5)$ \\
\hline Head/Neck & $4(2.0)$ \\
\hline Soft tissue & $6(3.0)$ \\
\hline Prostate/Bladder/Kidney/Testis & $29(14.5)$ \\
\hline Others & $11(5.5)$ \\
\hline Undergoing chemotherapy (yes) & $131(64.9)$ \\
\hline \multicolumn{2}{|l|}{ ECOG performance status } \\
\hline 0 & $7(3.5)$ \\
\hline 1 & $125(62.5)$ \\
\hline 2 & $55(27.5)$ \\
\hline 3 & $13(6.5)$ \\
\hline 4 & 0 \\
\hline \multicolumn{2}{|l|}{ KPS } \\
\hline $10-40$ & 0 \\
\hline 50 & $5(2.5)$ \\
\hline 60 & $16(8.0)$ \\
\hline 70 & $68(34.0)$ \\
\hline 80 & $86(43.0)$ \\
\hline 90 & $25(12.5)$ \\
\hline 100 & 0 \\
\hline \multicolumn{2}{|l|}{ Objective symptom assessment (yes) } \\
\hline Dyspnea & $65(32.5)$ \\
\hline Dysphagia & $11(5.5)$ \\
\hline Anorexia & $127(63.5)$ \\
\hline Edema & $41(20.5)$ \\
\hline Fatigue & $148(74.0)$ \\
\hline Weight loss & $63(31.5)$ \\
\hline
\end{tabular}

A prognostic model (score, 0-8.0) was developed based on the results of the multivariate analysis (Table 3 ). The score was calculated by summating the partial scores, ranging from 0 to 8.0. The cutoff point of 4.0 was selected because it showed the best sensitivity and specificity in predicting 3-month survival (Table 4). The sensitivity, specificity, and overall accuracy of the cutoff point of 4.0 were $75.6 \%, 83.4 \%$, and $81.8 \%$, respectively. The discriminating ability of the
Table 1. Continued

\begin{tabular}{|c|c|}
\hline Characteristic & No. $(\%)(n=200)$ \\
\hline \multicolumn{2}{|l|}{ Laboratory result } \\
\hline $\mathrm{WBC}(/ \mu \mathrm{L})$ & $6,045(1,300-26,830)$ \\
\hline Hemoglobin (g/dL) & $11.0(6.8-16.0)$ \\
\hline Platelet $\left(\times 10^{3} / \mu \mathrm{L}\right)$ & $240.5(234-728)$ \\
\hline Neutrophil (\%) & $65.8(29.1-94.0)$ \\
\hline Lymphocyte (\%) & $20.7(1.8-57.8)$ \\
\hline Total bilirubin (mg/dL) & $0.5(0.2-5.8)$ \\
\hline Albumin (mg/dL) & $3.8(2.0-4.8)$ \\
\hline AST (IU/L) & $25(9-523)$ \\
\hline ALT (IU / L) & $19(6-228)$ \\
\hline LDH (IU/L) & $222(56-4,000)$ \\
\hline Uric acid (mg/dL) & $4.9(1.1-15.5)$ \\
\hline $\mathrm{CRP}(\mathrm{mg} / \mathrm{dL})$ & $0.9(0.2-23.2)$ \\
\hline BUN (mg/dL) & $15(4-156)$ \\
\hline Creatinine $(\mathrm{mg} / \mathrm{dL})$ & $0.8(0.1-7.5)$ \\
\hline Triceps skin folds thickness (cm) & $1.7 \pm 0.8$ \\
\hline Mid-arm circumference (cm) & $26.5 \pm 3.8$ \\
\hline Survival (mo) & $7.6(0.2-36.8)$ \\
\hline $\begin{array}{l}\text { Time from the date of advanced } \\
\text { cancer diagnosis (mo) }\end{array}$ & $14.1(1.4-112.9)$ \\
\hline $\begin{array}{l}\text { Time from the date of being } \\
\text { diagnosed incurable (mo) }\end{array}$ & $13.9(1.4-112.9)$ \\
\hline
\end{tabular}

Values are presented as mean $\pm \mathrm{SD}$, number $(\%)$, or median (range). ALT, alanine transaminase; AST, aspartate transaminase; BUN, blood urea nitrogen; CRP, C-reactive protein; ECOG, Eastern Cooperative Oncology Group; KPS, Karnofsky performance status; $\mathrm{LDH}$, lactate dehydrogenase; SD, standard deviation; WBC, white blood cell.

prognostic model using the area under the ROC curve (AUC) was 0.88 (Fig. 1). The median survival for patients scoring 4 .0 points or more was 2.9 months (95\% CI, 2.1 to 4.3 ), compared with 11.3 months ( $95 \% \mathrm{CI}, 8.5$ to 13.4 ) for patients scoring less than 4.0 points $(\mathrm{p}<0.001)$ (Fig. 2).

\section{Discussion}

In this prospective cohort study, we developed a composite scoring system that can predict 3-month survival in oncology outpatients. Medical oncologists can identify patients with expected survival of less than 3 months using our model and may refer these patients to palliative care specialists in a timely manner. Our prognostic model comprises six independent predictors, including a poor ECOG performance status $(\geq 2)$, not undergoing chemotherapy, the presence of anorexia, a low lymphocyte level $(<12 \%)$, a high LDH level ( $\geq 300 \mathrm{IU} / \mathrm{L}$ ), and a low mid-arm circumference 
Table 2. Results of the univariate survival analysis

Variable

No.

$$
\begin{gathered}
\text { Age (yr) } \\
<65 \\
\geq 65
\end{gathered}
$$

Undergoing chemotherapy

No

Yes

Dyspnea

No 135

Yes

Dysphagia

$$
\text { No }
$$

Yes

Appetite loss

$$
\text { No }
$$

Yes

\section{Edema}

No

Yes

Fatigue

$$
\text { No }
$$

Yes

\section{Weight loss}

No

Yes

WBC $(/ \mu \mathrm{L})$

$<9,000$

$\geq 9,000$

\section{Hemoglobin (g/dL)}

$$
\geq 9.0
$$$$
<9.0
$$

Platelet $\left(\times 10^{3} / \mu \mathrm{L}\right)$

$$
<250
$$$$
\geq 250
$$

Neutrophil (\%)

$$
<80
$$$$
\geq 80
$$

Lymphocyte (\%)

$\begin{array}{rr}\geq 12 & 167 \\ <12 & 33\end{array}$

Total bilirubin $(\mathrm{mg} / \mathrm{dL})$

$$
<0.5
$$$$
\geq 0.5
$$

Albumin (mg/dL)

$$
\geq 3.5
$$$$
<3.5
$$

\section{AST (IU/L)}

$$
<40
$$

$$
\geq 40
$$

(Continued to the next page)
93

107

69

131

135
65

65

189

11

73

127

159

41

52

148

137

63

159

41

174

26

115

85

177

23

33

95

105

146

54

155

45

Median survival (mo) $(95 \%$ CI) p-value

$9.2(7.0-11.7)$

$6.7(5.8-8.0)$

$4.6(3.3-6.0)$

$9.8(7.9-13.3)$

$9.6(7.5-12.0)$

$5.8(3.1-6.7)$

$7.8(6.6-9.4)$

$2.9(1.3-25.1)$

$11.7(8.9-19.0)$

$<0.001$

$6.4(4.8-7.4)$

$8.4(7.0-10.8)$

$3.3(2.1-6.0)$

$13.3(8.2-22.1)$

$6.6(4.8-7.8)$

$8.9(6.9-10.9)$

0.071

$6.0(4.5-7.5)$

$8.4(6.9-11.4)$

$3.5(2.9-5.1)$

$8.0(6.7-10.3)$

$<0.001$

$4.2(2.1-6.3)$

$9.4(6.7-12.7)$

0.094

6.7 (4.7-7.9)

$7.9(6.7-9.8)$

0.002

$3.2(2.0-4.8)$

$8.4(7.2-10.9)$

$<0.001$

$4.0(2.3-4.8)$

$9.0(6.7-12.6)$

$6.7(4.8-7.9)$

$9.4(7.8-12.0)$ $<0.001$

$4.0(2.4-5.2)$

$8.3(6.9-11.3)$

$<0.001$

0.313

$<0.001$

$<0.001$

0.418

0.008

0.001

0.001

$4.4(2.6-7.0)$
0.098 
Table 2. Continued

\begin{tabular}{|c|c|c|c|}
\hline \multicolumn{4}{|c|}{ ALT (IU/L) } \\
\hline$<30$ & 155 & $7.4(6.0-9.0)$ & 0.918 \\
\hline$\geq 30$ & 45 & $8.0(4.9-12.0)$ & \\
\hline \multicolumn{4}{|c|}{ LDH (IU/L) } \\
\hline$<300$ & 155 & $9.5(7.9-11.7)$ & $<0.001$ \\
\hline$\geq 300$ & 44 & $3.0(2.1-3.9)$ & \\
\hline \multicolumn{4}{|c|}{ Uric acid (mg/dL) } \\
\hline$\geq 5.0$ & 98 & $8.4(6.4-11.7)$ & 0.266 \\
\hline$<5.0$ & 102 & $7.3(5.6-8.2)$ & \\
\hline \multicolumn{4}{|c|}{ CRP (mg/dL) } \\
\hline$<3.0$ & 153 & $9.4(7.4-11.7)$ & $<0.001$ \\
\hline$\geq 3.0$ & 46 & $3.3(2.1-5.8)$ & \\
\hline \multicolumn{4}{|c|}{ BUN (mg/dL) } \\
\hline$<15$ & 99 & $7.8(6.3-9.5)$ & 0.425 \\
\hline$\geq 15$ & 101 & $7.6(5.1-10.8)$ & \\
\hline \multicolumn{4}{|c|}{ Creatinine (mg/dL) } \\
\hline$>1.0$ & 136 & $7.5(5.8-8.9)$ & 0.340 \\
\hline$\leq 1.0$ & 64 & $7.9(6.3-14.4)$ & \\
\hline \multicolumn{4}{|c|}{ Triceps skin folds thickness (cm) } \\
\hline$\geq 0.8$ & 167 & $7.9(6.7-9.8)$ & 0.010 \\
\hline$<0.8$ & 26 & $4.4(2.9-7.6)$ & \\
\hline \multicolumn{4}{|c|}{ Mid-arm circumference $(\mathrm{cm})$} \\
\hline$\geq 23$ & 169 & $8.0(6.9-10.7)$ & $<0.001$ \\
\hline$<23$ & 24 & $2.6(1.7-4.3)$ & \\
\hline \multicolumn{4}{|c|}{ ECOG performance status } \\
\hline $0-1$ & 132 & $10.3(7.9-12.5)$ & $<0.001$ \\
\hline $2-3$ & 68 & $4.7(3.0-5.9)$ & \\
\hline \multicolumn{4}{|l|}{ KPS (\%) } \\
\hline 80-90 & 111 & $11.3(8.4-13.3)$ & $<0.001$ \\
\hline $10-70$ & 89 & $4.8(3.6-6.0)$ & \\
\hline
\end{tabular}

ALT, alanine transaminase; AST, aspartate transaminase; BUN, blood urea nitrogen; CI, confidence interval; CRP, C-reactive protein; ECOG, Eastern Cooperative Oncology Group; KPS, Karnofsky performance status; LDH, lactate dehydrogenase; WBC, white blood cell. a) ${ }^{\text {p-values }}$ were calculated by log-rank test.

$(<23 \mathrm{~cm})$. The accuracy of our model reached approximately $82 \%$ with a cutoff value of 4.0 .

Ideally, every advanced cancer patient should receive palliative care early in the disease trajectory [1-4]. However, there are many barriers to the early integration of palliative care into standard oncology care. Barriers may include limited access to palliative care services and specialists with highly variable palliative care infrastructures among cancer centers worldwide, heterogeneous referral patterns of individual physicians, and patient factors leading to delayed referrals $[11,13,15,16]$. Particularly, medical oncologists may focus on next chemotherapy options, thus they may often fail to recognize the appropriate timing of referral. The main advantage of our prognostic model is that it can accurately predict 3-month survival in advanced cancer patients. Thus it can facilitate palliative care referral consequently. In addition, it is an objective tool to minimize the interrater variability. Although it requires some laboratory data and a short training to measure mid-arm circumference, it is an easy-to-use scoring system that can be calculated by any health care provider. We expect that screening with our model can trigger medical oncologists to refer their patients to palliative care specialists before it is too late.

In advanced cancer patients, most prognostic models are developed for terminal cancer patients who are receiving palliative care as a sole focus of care. Externally validated and widely used models include the Palliative Performance Scale, Palliative Prognostic (PaP) Score, and Palliative Prognostic Index [17-19]. In South Korea, the Objective Prognostic Score is the only model validated and formally compared 
Table 3. A prognostic model based on the multivariate survival analysis

\begin{tabular}{lccccc} 
Variable & HR $(95 \%$ CI $)$ & $\boldsymbol{\beta}$ & SE & p-value $^{\text {a) }}$ & Partial score $^{\text {b) }}$ \\
Undergoing chemotherapy (yes vs. no) & $1.98(1.39-2.84)$ & 0.69 & 0.18 & $<0.001$ & 1 \\
Anorexia (no vs. yes) & $1.88(1.32-2.68)$ & 0.63 & 0.18 & $<0.001$ & 1 \\
Lymphocyte $(\geq 12 \%$ vs. $<12 \%)$ & $2.41(1.57-3.68)$ & 0.88 & 0.22 & $<0.001$ & 1.5 \\
LDH $(<300 \mathrm{IU} / \mathrm{L}$ vs. $\geq 300 \mathrm{IU} / \mathrm{L})$ & $3.26(2.23-4.77)$ & 1.18 & 0.19 & $<0.001$ & 2 \\
Mid-arm circumference $(\geq 23 \mathrm{~cm}$ vs. $<23 \mathrm{~cm})$ & $2.78(1.71-4.53)$ & 1.02 & 0.25 & $<0.001$ & 1.5 \\
ECOG performance status (0-1 vs. 2-3) & $1.64(1.14-2.37)$ & 0.50 & 0.19 & 0.008 & 1 \\
\hline
\end{tabular}

CI, confidence interval; ECOG, Eastern Cooperative Oncology Group; HR, hazard ratio; LDH, lactate dehydrogenase; SE, standard error.

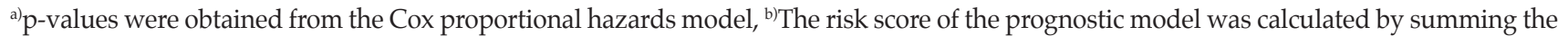
partial scores of undergoing chemotherapy, anorexia, lymphocyte level, LDH level, mid-arm circumference and ECOG performance status (range, 0-8.0).

Table 4. Accuracy of the prognostic model to predict 3-month survival according to different cutoff points

\begin{tabular}{lccccc}
$\begin{array}{l}\text { Prognostic } \\
\text { score }\end{array}$ & Sensitivity (\%) & Specificity (\%) & $\begin{array}{c}\text { Positive } \\
\text { predictive value (\%) }\end{array}$ & $\begin{array}{c}\text { Negative } \\
\text { predictive value }(\%)\end{array}$ & \begin{tabular}{c} 
Accuracy $(\%)$ \\
\hline 6
\end{tabular} \\
\hline 5.5 & 22.0 & 100 & 100 & 82.5 & 83.3 \\
\hline 5 & 34.2 & 96.7 & 73.7 & 84.4 & 83.3 \\
\hline 4.5 & 41.5 & 95.4 & 70.8 & 85.7 & 83.9 \\
\hline 4 & 58.5 & 90.1 & 58.5 & 88.9 & 83.3 \\
\hline 3.5 & 75.6 & 83.4 & 55.4 & 92.6 & 78.6 \\
\hline 3 & 80.5 & 78.2 & 50.0 & 93.7 & 65.1 \\
\hline 2.5 & 97.6 & 56.3 & 37.7 & 98.8 & 63.0 \\
\hline 2 & 97.6 & 53.6 & 36.4 & 100 & 40.1 \\
\hline
\end{tabular}

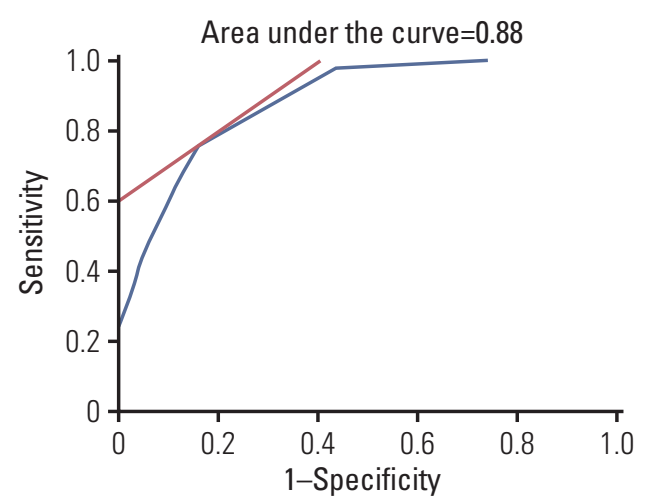

Fig. 1. Receiver operating characteristic curve of prognostic score.

with other validated scales [20]. Although some studies attempted to validate the $\mathrm{PaP}$ score in advanced cancer patients receiving chemotherapy $[21,22]$, the interpretation may be limited because this model was initially developed in terminal cancer patients. Few studies have focused on patients who are still receiving anticancer treatment [2325]. One study predicted 2-month survival in hospitalized

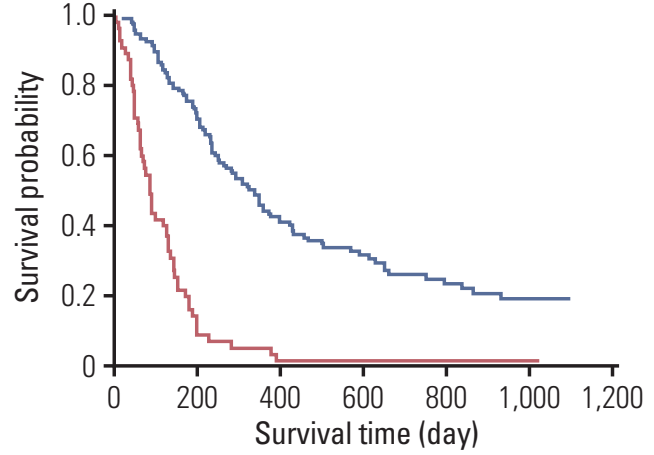

Fig. 2. Kaplan-Meier survival curves according to a cutoff point of 4.0. Patients with a risk score $\geq 4.0$ (red) had a median survival of 2.9 months (95\% confidence interval [CI], 2.1 to 4.3 ), whereas patients with a risk score $<4.0$ (blue) had a median survival of 11.3 months (95\% CI, 8.5 to 13.4). The difference in survival was significant $(p<0.001)$. It was calculated by the log-rank test.

advanced cancer patients, and the KPS, number of metastatic sites, low serum albumin, and high LDH level were independent prognostic factors [23]. Another study enrolled patients referred to a radiation oncology clinic, and the KPS, 
location of primary cancer (breast versus nonbreast), site of metastatic disease (bone only versus others), fatigue, anorexia, and dyspnea were significant factors [24]. The last study enrolled patients treated at a French medical oncology clinic who had failed to at least one line of chemotherapy. In this study, a poor ECOG performance status $(>1)$, low lymphocyte count, high LDH level, high interleukin 6 level, low serum albumin level, and low platelet count were identified as significant prognostic factors [25]. More recently, some studies have attempted to predict prognosis in ambulatory cancer patients $[26,27]$. The PRONOPALL study could categorize 262 patients into three distinct prognostic groups based on four factors including ECOG performance status, the number of metastatic sites, serum albumin, and LDH [26]. The Barretos Prognostic Nomogram included 497 Brazilian patients at the time of the first consultation to outpatient palliative care clinic [27]. Many patients were still receiving anticancer treatment at the time of consultation, and five parameters including sex, presence of distant metastasis, KPS, white blood cell count, and serum albumin concentration could discriminate patients into three distinct prognostic groups. The AUC for 90-day survival was 0.74. Similar to these studies, our study identified a poor ECOG performance status, low lymphocyte count, high LDH level, and anorexia as independent prognostic factors. Our study may be distinct from most of the above-mentioned studies in that all our patients were medical oncology outpatients and all of them had received treatment in the modern targeted therapy and immunotherapy era. Although our model needs external validation, the AUC for 3-month survival was high, which was 0.88 .

In the present study, whether the patient was undergoing chemotherapy or not was an independent prognostic factor according to the multivariate analysis. Unfortunately, we were unable to clarify the exact reasons for not undergoing systemic chemotherapy in these patients. However, we assume that a considerable number of ambulatory medical oncology patients with limited chemotherapy options had participated in this study just before or at the time of formal palliative care consultations. Many patients seek clinical trials or new chemotherapeutic agents not covered by government health insurance in our institution before considering palliative care referrals since there are increased availabilities of genotype-directed targeted agents and immune checkpoint inhibitors. A similar phenomenon was described in a study that analyzed the later-line use of immune checkpoint inhibitors in patients with advanced non-small cell lung cancer with impaired ECOG performance status $(\geq 2)$. In that study, patients with impaired performance status had significantly shorter survival after using immune checkpoint inhibitors (4.5 months in patients with ECOG performance status $\geq 2$ and 14.3 months in those with performance status 0 or 1 ), and receipt of an immune checkpoint inhibitor near death was associated with lower hospice use and an increased risk of death in the acute care hospital [12]. Medical oncologists should always be aware that impaired performance status and / or a later-line chemotherapy are red flags for advanced cancer patients who are not yet formally referred to palliative care specialists.

Notably, mid-arm circumference was identified as a significant prognostic factor in this study. Growing evidence suggests that ongoing loss of skeletal muscle mass is an important part of cancer cachexia, and may predict survival in cancer patients [28]. Considering the complexity of assessing muscle depletion, we attempted to use mid-arm circumference and triceps skinfold thickness as surrogate markers. Both were statistically significant prognostic factors in the univariate analysis, but mid-arm circumference remained in the final model after the multivariate analysis. In a prospective study of 4,107 men aged 60 to 79 years, a low mid-arm muscle circumference (MAMC) level was significantly associated with poor survival [29]. MAMC was calculated from mid-arm circumference and triceps skinfold thickness. In another study, mid-arm muscle area predicted mortality in stable chronic obstructive pulmonary disease patients [30]. In our study, we could not calculate the MAMC because there were no data on the average value of MAMC in Koreans. Further studies may be required to validate and further investigate our findings. However, mid-arm circumference is a simple and inexpensive anthropometric measure that can be widely accepted in busy oncology clinics. We believe that mid-arm circumference can provide useful prognostic information.

Our study has several limitations. First, it was conducted at a single tertiary cancer center in a heterogeneous group of patients in South Korea. Therefore, additional studies are required to examine the feasibility of applying this model in different settings worldwide. Second, some important factors including the clinician prediction of survival were not included in the analysis. However, the inclusion criteria were based on the oncologists' prediction of 1-year survival, and we identified highly significant factors despite various types of cancer and various states of disease in participants. Last, external validation of the prognostic model has not yet been performed.

In conclusion, our study demonstrated that the prognostic model using ECOG performance status, chemotherapy status, the presence of anorexia, lymphocyte levels, LDH levels, and mid-arm circumference can predict 3-month survival in medical oncology outpatients. We expect this model to trigger medical oncologists to refer patients to palliative care specialists before it is too late. 


\section{Ethical Statement}

Written informed consent was obtained from each patient before enrollment. The protocol was approved by the Institutional Review Board (IRB) of Seoul National University Bundang Hospital (IRB number: B-1601/332-302).

\section{Author Contributions}

Conceived and designed the analysis: Kim YJ, Suh SY, Kang B. Collected the data: Kim YJ, Suh SY, Kang B, Lee SW, Suh KJ, Kim JW (Jin Won Kim), Kim SH, Kim JW (Ji-Won Kim), Lee KW, Kim $\mathrm{JH}$, Lee JS.

Contributed data or analysis tools: Kim YJ, Hiratsuka Y, Suh SY, Kang B, Lee SW, Suh KJ, Kim JW (Jin Won Kim), Kim SH, Kim JW (Ji-Won Kim), Lee KW, Kim JH, Lee JS.

Performed the analysis: Kim YJ, Hiratsuka Y, Suh SY, Ahn HY.
Wrote the paper: Kim YJ, Hiratsuka Y, Suh SY, Kang B, Lee SW, Ahn HY, Suh KJ, Kim JW (Jin Won Kim), Kim SH, Kim JW (Ji-Won Kim), Lee KW, Kim JH, Lee JS.

\section{Conflicts of Interest}

Conflict of interest relevant to this article was not reported.

\section{Acknowledgments}

The authors have declared no conflicts of interest. This work was supported by the National Research Foundation of Korea (NRF) grant funded by the Korean government (Ministry of Science, Informatics, Communication and Technology; No. 2015R1C1A2A01053357). We thank the participating patients and clinical research nurses Jin Suk Kim and Esther Jeon for supporting this study.

\section{References}

1. Bakitas M, Lyons KD, Hegel MT, Balan S, Brokaw FC, Seville $\mathrm{J}$, et al. Effects of a palliative care intervention on clinical outcomes in patients with advanced cancer: the Project ENABLE II randomized controlled trial. JAMA. 2009;302:741-9.

2. Temel JS, Greer JA, Muzikansky A, Gallagher ER, Admane S, Jackson VA, et al. Early palliative care for patients with metastatic non-small-cell lung cancer. N Engl J Med. 2010;363:73342.

3. Zimmermann C, Swami N, Krzyzanowska M, Hannon B, Leighl N, Oza A, et al. Early palliative care for patients with advanced cancer: a cluster-randomised controlled trial. Lancet. 2014;383:1721-30.

4. Ferrell BR, Temel JS, Temin S, Alesi ER, Balboni TA, Basch EM, et al. Integration of palliative care Into standard oncology vare: American Society of Clinical Oncology clinical practice guideline update. J Clin Oncol. 2017;35:96-112.

5. NHPCO facts and figures [Internet]. Alexandria, VA: National Hospice and Palliative Care Organization; 2020 [cited 2020 Nov 29]. Available from: https://www.nhpco.org/wp-content/uploads/NHPCO-Facts-Figures-2020-edition.pdf.

6. Hospice UK. Hospice care in the UK [Internet]. London: Hospice UK; 2016 [cited 2020 Nov 29]. Available from: https:// www.hospiceuk.org/docs/default-source/What-We-Offer/ publications-documents-and-files/hospice-care-in-theuk-2016.pdf?sfvrsn=0.

7. National Cancer Center, Hospice and Palliative Care [Internet]. Goyang: National Cancer Center; 2020 [cited 2020 Nov 29]. Available from: http://hospice.cancer.go.kr/pain/stat.do? menu_no $=634 \&$ brd_mgrno=

8. Dalal S, Bruera S, Hui D, Yennu S, Dev R, Williams J, et al. Use of palliative care services in a tertiary cancer center. Oncologist. 2016;21:110-8.

9. Hui D, Kim SH, Kwon JH, Tanco KC, Zhang T, Kang JH, et al. Access to palliative care among patients treated at a compre- hensive cancer center. Oncologist. 2012;17:1574-80.

10. Hui D, Kim SH, Roquemore J, Dev R, Chisholm G, Bruera E. Impact of timing and setting of palliative care referral on quality of end-of-life care in cancer patients. Cancer. 2014;120:1743-9.

11. Hui D, Kilgore K, Park M, Liu D, Kim YJ, Park JC, et al. Pattern and predictors of outpatient palliative care referral among thoracic medical oncologists. Oncologist. 2018;23:1230-5.

12. Petrillo LA, El-Jawahri A, Nipp RD, Lichtenstein MR, Durbin SM, Reynolds KL, et al. Performance status and end-of-life care among adults with non-small cell lung cancer receiving immune checkpoint inhibitors. Cancer. 2020;126:2288-95.

13. Bruera E, Hui D. Conceptual models for integrating palliative care at cancer centers. J Palliat Med. 2012;15:1261-9.

14. Cho JH, Yan US. Standard values for nutritional assessment by anthropometry in healthy Korean adults. Korean J Med. 1999;56:560-8.

15. Kruser TJ, Kruser JM, Gross JP, Moran M, Kaiser K, Szmuilowicz E, et al. Medical oncologist perspectives on palliative care reveal physician-centered barriers to early integration. Ann Palliat Med. 2020;9:2800-8.

16. Ehrlich BS, Movsisyan N, Batmunkh T, Kumirova E, Borisevich $\mathrm{MV}$, Kirgizov $\mathrm{K}$, et al. Barriers to the early integration of palliative care in pediatric oncology in 11 Eurasian countries. Cancer. 2020;126:4984-93.

17. Anderson F, Downing GM, Hill J, Casorso L, Lerch N. Palliative performance scale (PPS): a new tool. J Palliat Care. 1996;12:5-11.

18. Pirovano M, Maltoni M, Nanni O, Marinari M, Indelli M, Zaninetta $\mathrm{G}$, et al. A new palliative prognostic score: a first step for the staging of terminally ill cancer patients. Italian Multicenter and Study Group on Palliative Care. J Pain Symptom Manage. 1999;17:231-9.

19. Morita T, Tsunoda J, Inoue S, Chihara S. The Palliative Prog- 
nostic Index: a scoring system for survival prediction of terminally ill cancer patients. Support Care Cancer. 1999;7:128-33.

20. Suh SY, Choi YS, Shim JY, Kim YS, Yeom CH, Kim D, et al. Construction of a new, objective prognostic score for terminally ill cancer patients: a multicenter study. Support Care Cancer. 2010;18:151-7.

21. Glare PA, Eychmueller S, McMahon P. Diagnostic accuracy of the palliative prognostic score in hospitalized patients with advanced cancer. J Clin Oncol. 2004;22:4823-8.

22. Tassinari D, Montanari L, Maltoni M, Ballardini M, Piancastelli A, Musi M, et al. The palliative prognostic score and survival in patients with advanced solid tumors receiving chemotherapy. Support Care Cancer. 2008;16:359-70.

23. Barbot AC, Mussault P, Ingrand P, Tourani JM. Assessing 2-month clinical prognosis in hospitalized patients with advanced solid tumors. J Clin Oncol. 2008;26:2538-43.

24. Chow E, Abdolell M, Panzarella T, Harris K, Bezjak A, Warde $\mathrm{P}$, et al. Predictive model for survival in patients with advanced cancer. J Clin Oncol. 2008;26:5863-9.

25. Tredan O, Ray-Coquard I, Chvetzoff G, Rebattu P, Bajard A, Chabaud $S$, et al. Validation of prognostic scores for survival in cancer patients beyond first-line therapy. BMC Cancer.
2011;11:95.

26. Bourgeois H, Grude F, Solal-Celigny P, Dupuis O, Voog E, Ganem $\mathrm{G}$, et al. Clinical validation of a prognostic tool in a population of outpatients treated for incurable cancer undergoing anticancer therapy: PRONOPALL study. Ann Oncol. 2017;28:1612-7.

27. Paiva CE, Paiva BS, de Paula Pantano N, Preto DD, de Oliveira $\mathrm{CZ}$, Yennurajalingam $\mathrm{S}$, et al. Development and validation of a prognostic nomogram for ambulatory patients with advanced cancer. Cancer Med. 2018;7:3003-10.

28. Martin L, Birdsell L, Macdonald N, Reiman T, Clandinin MT, McCargar LJ, et al. Cancer cachexia in the age of obesity: skeletal muscle depletion is a powerful prognostic factor, independent of body mass index. J Clin Oncol. 2013;31:1539-47.

29. Wannamethee SG, Shaper AG, Lennon L, Whincup PH. Decreased muscle mass and increased central adiposity are independently related to mortality in older men. Am J Clin Nutr. 2007;86:1339-46.

30. Soler-Cataluna JJ, Sanchez-Sanchez L, Martinez-Garcia MA, Sanchez PR, Salcedo E, Navarro M. Mid-arm muscle area is a better predictor of mortality than body mass index in COPD. Chest. 2005;128:2108-15. 